\title{
Subdural Hematoma Mimics Epidural Hematoma: A Case Report
}

\author{
Gholamreza Faridaalaee $^{1^{*}}$, Ali Pashapour ${ }^{2}$, Fatemeh Keigobadi Khajeh ${ }^{3}$ \\ ${ }^{1}$ Department of Emergency Medicine, Faculty of Medicine, Urmia University of Medical Sciences, Urmia, Iran. \\ ${ }^{2}$ Department of Neurosurgery, Faculty of Medicine, Urmia University of Medical Sciences, Urmia, Iran. \\ ${ }^{3}$ Department of Emergency Medicine, Tabriz University of Medical Sciences, Tabriz, Iran.
}

\section{ABSTRACT}

Introduction: Traumatic brain injuries (TBI) are complex injuries with a broad range of symptoms and disabilities. TBI include epidural hematoma (EDH), subdural hematoma (SDH), intracerebral hemorrhage, subarachnoid hemorrhage (SAH), diffuse axonal injury, and brain contusion. Case Description: The patient was a 22-year-old man referred to the emergency department of Imam Khomeini hospital, Urmia, Iran, suffering from headache. Headache severity was 8 out of 10 (according to visual analog scale). The patient was fully alert and Glasgow coma scale was 15 . Brain CT scan showed EDH and blood that filled the sylvain fissure. Results: It was revealed during surgery that patient was suffering from SDH with bleeding in a chronic arachnoid cyst. Conclusion: SDH is the collection of blood in the space between the dura and subarachnoid layer and originating from veins. SDH is more common in alcoholic and older patients. In rare cases, blood is collected arachnoid cyst and is seen like SAH.

\section{Key words:}

1. Hematoma, Subdural 2. Hematoma, Epidural, Cranial

3. Brain

4. Subarachnoid

Hemorrhage

* Corresponding Author: Gholamreza Faridaalaee

E-mail:aalae1391@gmail.com 


\section{خونريزى زير سخت شامهاى شبيه خونريزى إيبدورال: يك گزارش مورد}

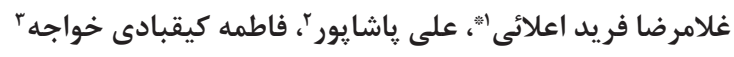

$$
\text { 'كروه طب اورزانس، دانشكده يزشكى، دانشكاه علوم يزشكى اروميه، اروميه، ايران. }
$$

rكروه جراحى مغز و اعصاب، دانشكده يزشكى، دانشَاه علوم يزشكى اروميه، اروميه، ايران.

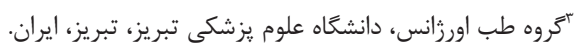

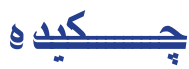

مقدمه: آسيبهاى مغزى ناشى از ضربه، آسيبهاى ييجيده با يك محدوده وسيعى از علايمى و ناتوانىها

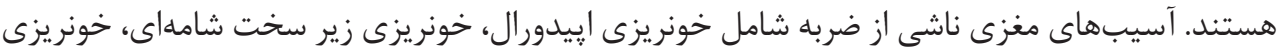

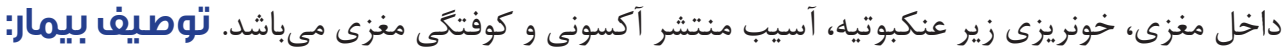

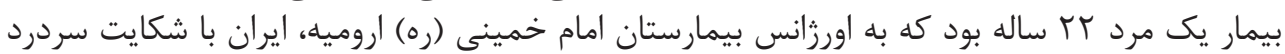

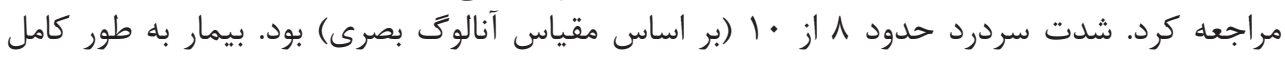

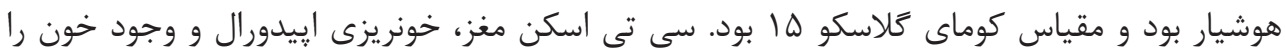

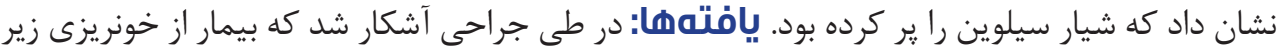

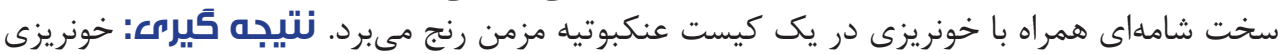

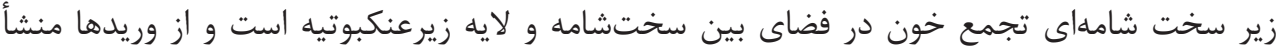

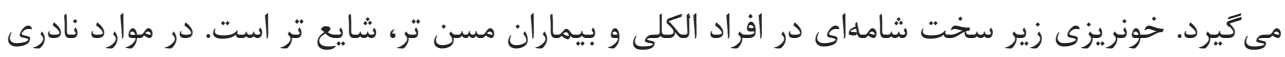

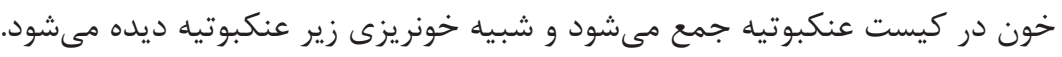

كليد وازهها:

ا. خونريزى زير سخت شامهاى

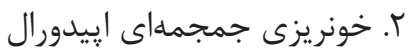

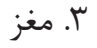
أ. خونريزى زير عنكبوتيه 


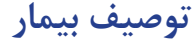

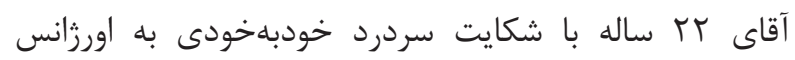

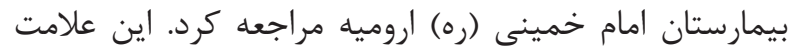

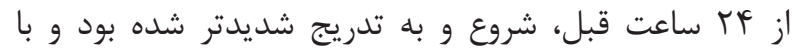

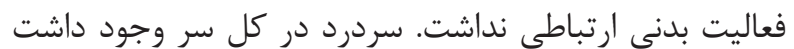

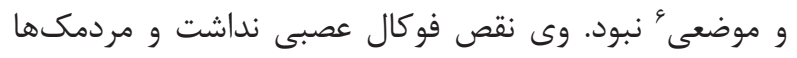

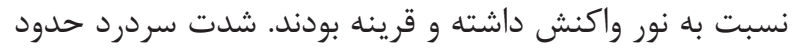

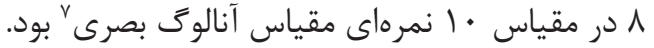

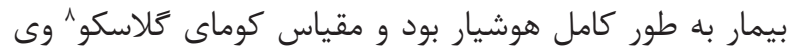

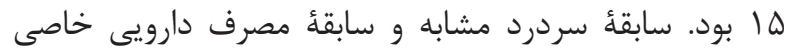

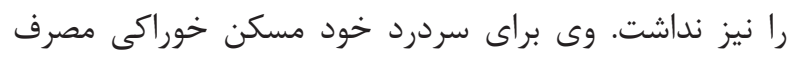

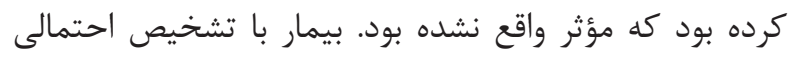

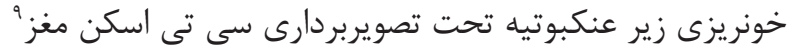

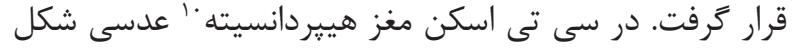

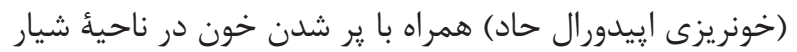

$$
\text { سيلوين مشهود بود (تصوير ( ). }
$$

بيمار با تشخيص خونريزى إيدورال حاد، كرانيوتومى شد. در

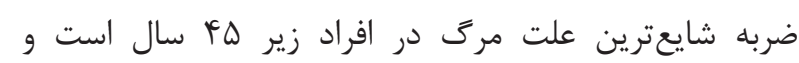

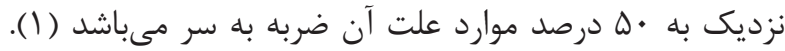

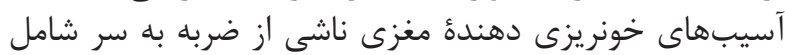

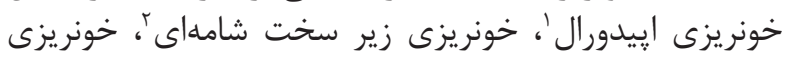

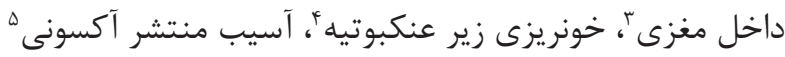

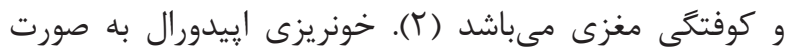

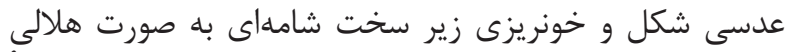

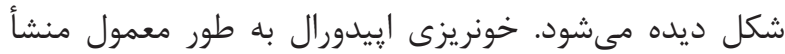

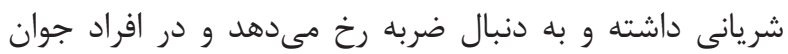

$$
\text { بيشتر ديده مىشود (r). }
$$

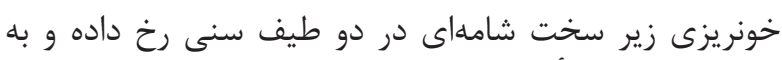

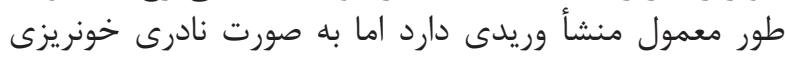

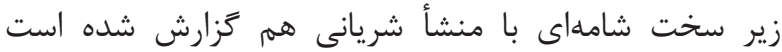

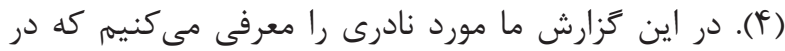

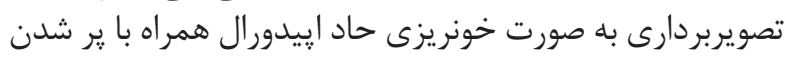

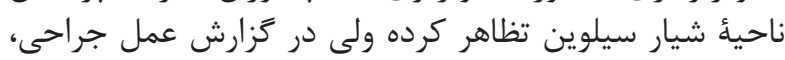

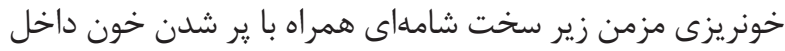
كيست از قبل تشكيل شده عنكبوتيه دئ ديده شداي

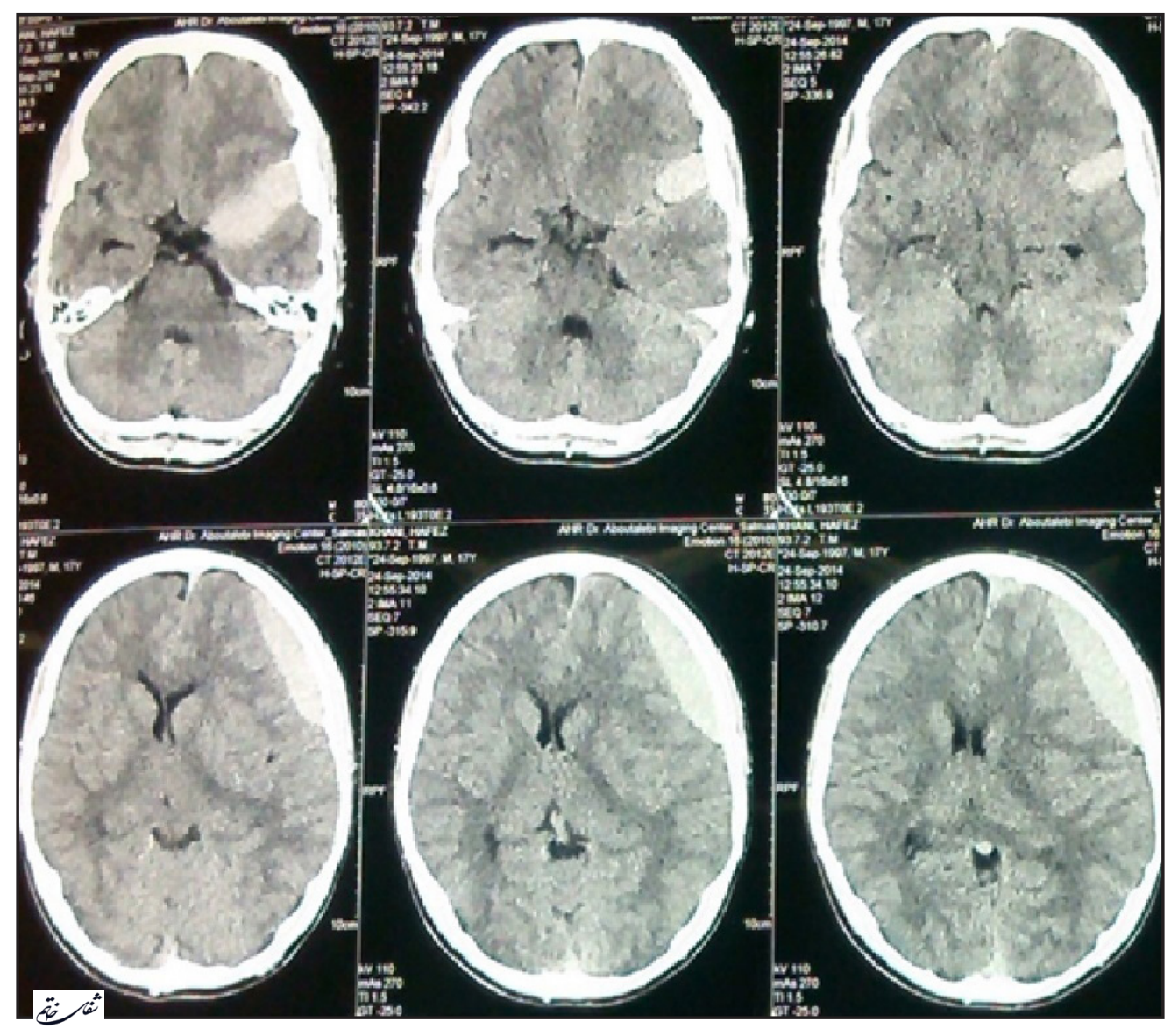

تصوير ا- سى تى اسكن سر بيمار. خونريزى عدسى شكل با بر شدن خون در شيار سيلوين مطرح كنندة خونريزى إيدورال مى باشد.

'Epidural hematoma (EDH)

${ }^{2}$ Subdural hematoma (SDH)

${ }^{3}$ Intracerebral hemorrhage (ICH)

${ }^{4}$ Subarachnoid hemorrhage (SAH)

${ }^{5}$ Diffuse axonal injury (DAI)
${ }^{6}$ Localized

${ }^{7}$ Visual analog scale (VAS)

${ }^{8}$ Glasgow coma scale (GCS)

${ }^{9}$ Brain CT scan

${ }^{10}$ Hyperdensity 

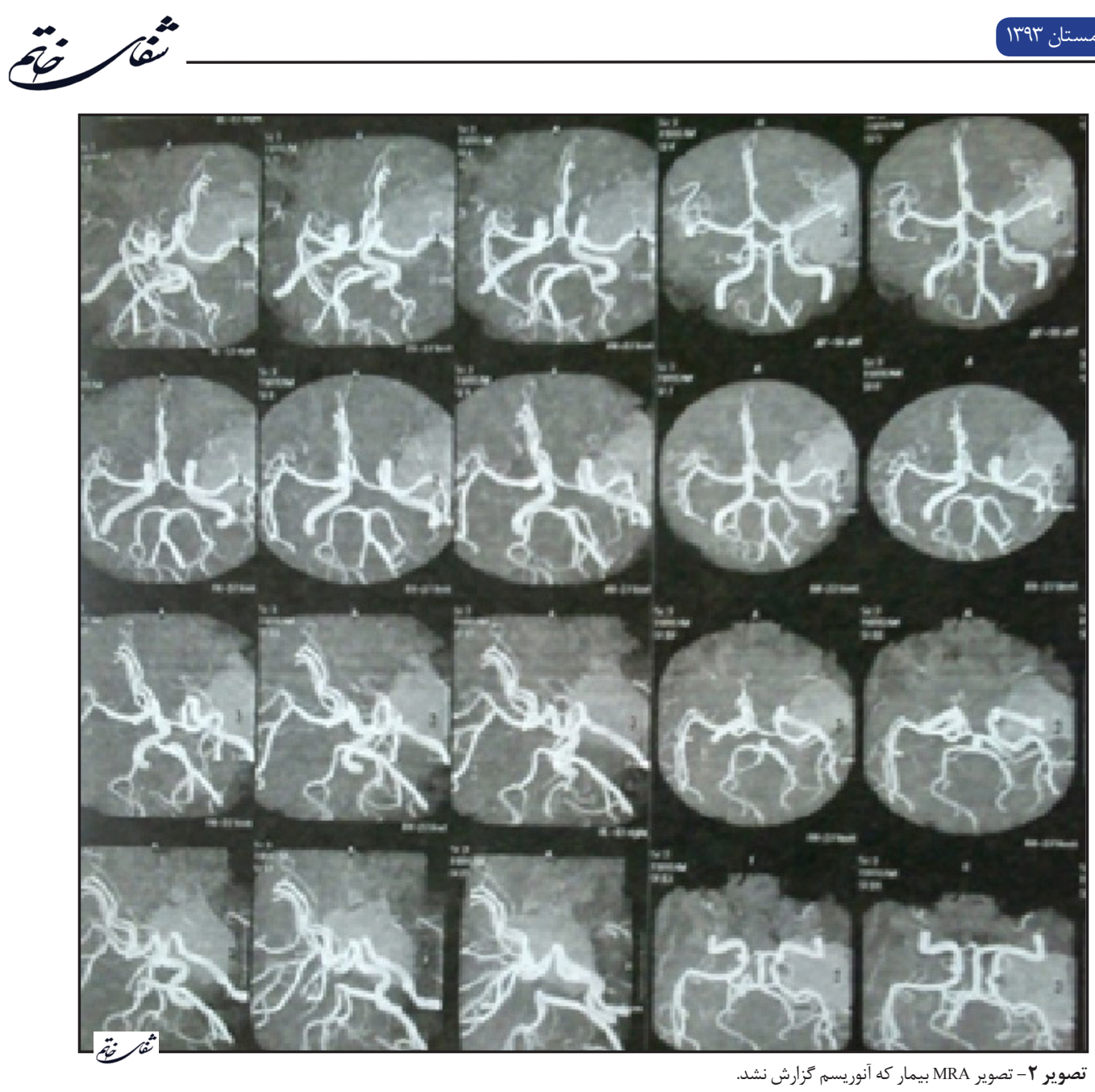

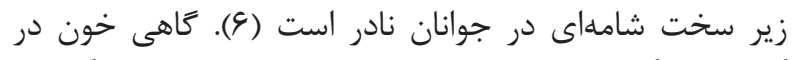

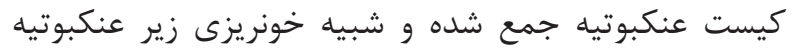

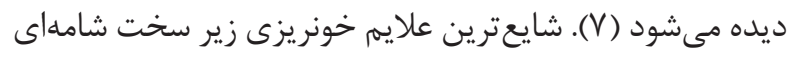

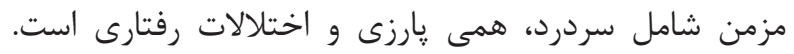

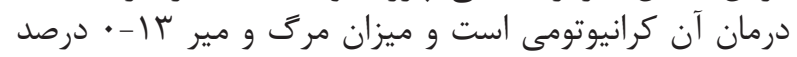

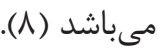

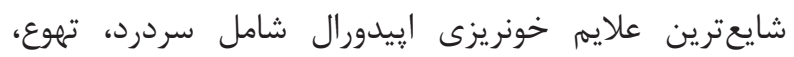

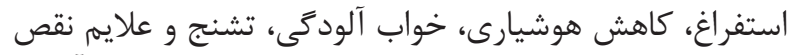

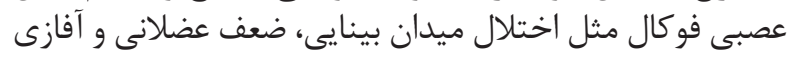

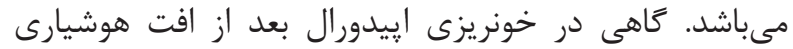

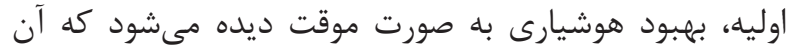

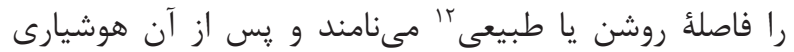

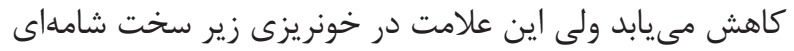

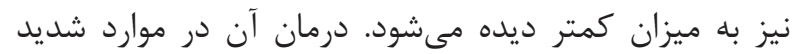

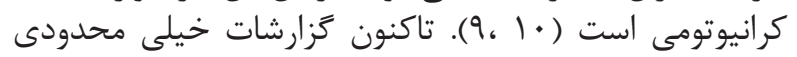

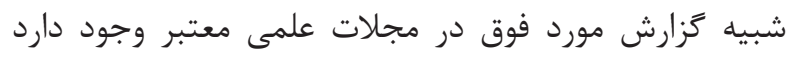

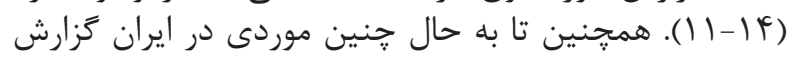
نشده بود.

$$
\text { تشكر و قدردانى }
$$

از كليهُ يرسنل بخش اورزانس و جراحى مغز و اعصاب بيمارستان

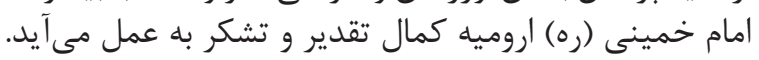

صورتى كه خونريزى إيدورال مشاهده نشد. با توجه به تصاوير

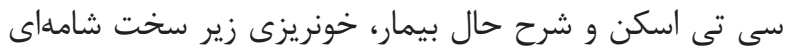

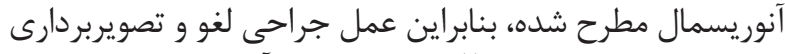

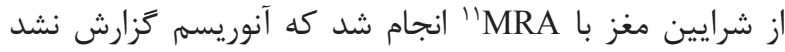

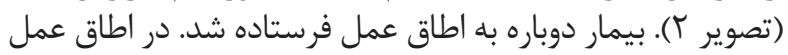

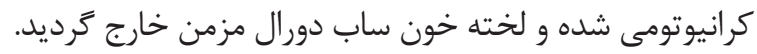

بافتهها نتايج عمل جراحى نشان داد كه بيمار خونريزى مغزى مزمن زير

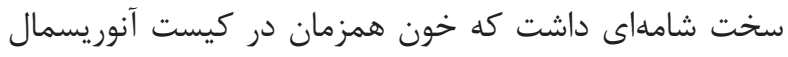

$$
\text { هم جمع شده بود. }
$$

در اين ززارش، ما موردى جالب از خونريزى زير سخت شامهاى

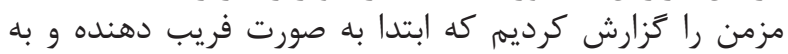

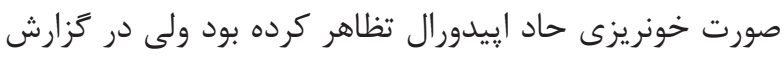

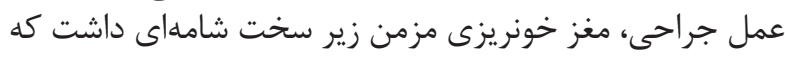
در كيست عنكبوتيه هم خون جمريز جمع شده شئ بود.

در خونريزى زير سخت شامهاى به طور معمول خون با منشأ

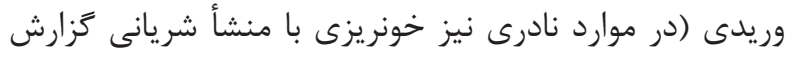

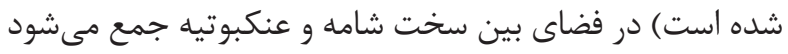

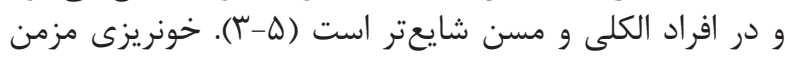

${ }^{11}$ Magnetic resonance angiography (MRA)

${ }^{12}$ Lucid interval 
1. MacKenzie EJ. Epidemiology of injuries: current trends and future challenges. Epidemiol Rev. 2000; 22(1): 112-9.

2. Tallon JM, Ackroyd-Stolarz S, Karim SA, Clarke DB. The epidemiology of surgically treated acute subdural and epidural hematomas in patients with head injuries: a population-based study. Can J Surg. 2008; 51(5): 339-45.

3. Markwalder TM. Chronic subdural hematomas: a review. J Neurosurg. 1981; 54(5): 637-45.

4. Koç RK, Paşaoğlu A, Kurtsoy A, Oktem IS, Kavuncu I. Acute spontaneous subdural hematoma of arterial origin: a report of five cases. Surg Neurol. 1997; 47(1): 9-11.

5. Haines DE, Harkey HL, al-Mefty O. The "subdural" space: a new look at an outdated concept. Neurosurgery. 1993; 32(1): 111-20.

6. Gelabert-Gonzalez M, Frieiro-Dantas C, SerramitoGarcia R, Diaz-Cabanas L, Aran-Echabe E, Rico-Cotelo $\mathrm{M}$, et al. Chronic subdural hematoma in young patients. Neurocirugia (Astur). 2013; 24(2): 63-9.

7. Kalz F, Klein A, Huisman TA. What is your diagnosis? Acute hemorrhage into known subarachnoid cyst. Praxis (Bern 1994). 2007; 96(5): 133-5.

8. Sousa EB, Brandão LF, Tavares CB, Borges IB, Neto NG, Kessler IM. Epidemiological characteristics of 778 patients who underwent surgical drainage of chronic subdural hematomas in Brasilia, Brazil. BMC Surg. 2013; 13: 5. doi: 10.1186/1471-2482-13-5.

9. Bratton SL, Chestnut RM, Ghajar J, McConnell Hammond FF, Harris OA, Hartl R, et al. Guidelines for the management of severe traumatic brain injury. IX. Cerebral perfusion thresholds. J Neurotrauma. 2007; 24 Suppl 1: S59-64.

10. Servadei F. Prognostic factors in severely head injured adult patients with epidural haematoma's. Acta Neurochir (Wien). 1997; 139(4): 273-8.

11. Hurvitz SA, Stone LR, Keenan MAE, Waters RL. Acute subdural haematoma mimicking an epidural haematoma on a CT scan. Brain Inj. 1989; 3(1): 63-5.

12. Miki S, Fujita K, Katayama W, Sato M, Kamezaki T, Matsumura A, et al. Encapsulated acute subdural hematoma mimicking acute epidural hematoma on computed tomography. Neurol Med Chir (Tokyo). 2012; 52(11): 826-8.

13. Prieto R, Pascual JM, Subhi-Issa I, Yus M. Acute epidural-like appearance of an encapsulated solid nonorganized chronic subdural hematoma. Neurol Med Chir (Tokyo). 2010; 50(11): 990-4

14. Su I-C, Wang K-C, Huang S-H, Li C-H, Kuo L-T, Lee J-E, et al. Differential CT features of acute lentiform subdural hematoma and epidural hematoma. Clin Neurol Neurosurg. 2010; 112(7): 552-6. 